\title{
Use of gelatin-thrombin matrix haemostatic sealant in neurosurgery: Anaesthetic implications and review of literature
}

\author{
Puneet Khanna, Charu Mahajan, Priyanka Gupta, Sujoy Banik, Bikash R Ray, Girija Prasad Rath
}

\begin{abstract}
The topical haemostatic agents have been developed to be used as adjunctive measures to promote haemostasis. These include bone wax, absorbable gel sponges, microfibrillar collagen, oxidised regenerated cellulose, gelatin sponges with thrombin, gelatin-thrombin matrix sealant or fibrin sealants. Gelatin-thrombin matrix sealant is a mixture of a bovine-derived gelatin matrix and human-derived thrombin component that are mixed together at the time of use. This agent has been found to be more effective haemostat than thrombin-soaked gelatine sponges. The possible adverse effects of this can be transmission of diseases from human or bovine sources, allergic reactions, thromboembolism, disseminated intravascular coagulopathy (DIC), perilesional oedema, and compression of neural tissue. Although it is used routinely in the operating room, there is little literature available on the perioperative implications with use of intraoperative gelatin-thrombin matrix sealant. Here, we present clinical report of 20 neurosurgical patients where the sealant was used and literature in view of current evidence has been reviewed.
\end{abstract}

Key words: Anaesthesia, haemostatic sealant, intraoperative bleeding, neurosurgery

\section{INTRODUCTION}

Bleeding from operative site during intracranial surgery obscures the visualisation of important structures. It not only complicates the procedure but also increases the requirement of blood transfusion. Moreover, during microneurosurgery, the hampered vision may lead to iatrogenic trauma to adjacent vital structures. Perfect haemostasis is especially important in neurosurgical patients, as any haematoma inside a closed cranial cavity can have devastating consequences. Bleeding from identifiable vessels can be controlled by means of mechanical compression by cottonoids, use of cautery or

\begin{tabular}{|l|l|}
\hline \multicolumn{2}{|c|}{ Access this article online } \\
\hline Quick Response Code: & Website: \\
\hline & www.??? \\
\cline { 2 - 2 } & \\
\hline
\end{tabular}

application of haemostatic clips. However, haemostasis is difficult to be achieved in case of diffuse capillary bleeding or in conditions where use of cautery may cause vessel damage and thermal injury to adjacent neural tissue. The topical haemostatic agents have been developed and used as adjunctive measures to promote haemostasis for nearly 75 years. They include bone wax, absorbable gel sponges, microfibrillar collagen, oxidised regenerated cellulose, gelatin sponges with thrombin, gelatin-thrombin matrix sealant or fibrin sealants.

Gelatin-thrombin matrix sealant is a mixture of a bovine-derived gelatin matrix and human-derived thrombin component that are mixed together at time of use. Food and Drug administration (FDA) approved FloSeal for the first time in 1999 for this purpose. This agent has been found to be more effective haemostat than thrombin-soaked gelatine sponges. ${ }^{[1]}$ The possible adverse effects of this can be transmission of diseases from human or bovine sources, allergic reactions, perilesional oedema, compression of neural tissue, thromboembolism, development of factor

Department of Neuroanaesthesiology, All India Institute of Medical Sciences, New Delhi, India

Address for correspondence:

Dr. Charu Mahajan, Department of Neuroanaesthesiology, Neurosciences Centre; $6^{\text {th }}$ Floor/ Room \# 9, All India Institute of Medical Sciences, New Delhi - 110 029, India. E-mail: charushrikul@gmail.com 
$\mathrm{V}$ antibodies or even disseminated intravascular coagulopathy (DIC). Although it is used routinely in the operating room, there is little literature available on the perioperative implications with use of intraoperative gelatin-thrombin matrix sealant. Here, we present clinical report of 20 neurosurgical patients where the sealant was used and literature in view of current evidence has been reviewed.

\section{MATERIALS AND METHODS}

This retrospective study was carried out to evaluate the safety, efficacy, post-operative complications and outcome in neurosurgical patientsin whom gelatin-thrombin matrix sealant was used for achieving topical haemostasis during the surgical procedure. The information on FloSeal use in these patients was retrieved from the nurse's records. Thereafter, the case files of these patients were obtained from the record section. The information regarding demographic details, presenting symptoms, associated comorbidities, drug treatment, preoperative investigations, anaesthetic technique, intraoperative blood loss, fluids infused, blood and blood product transfusion, postoperative investigations and surgical outcome were collected from anaesthesia record sheet, case files and discharge notes.

\section{RESULTS}

Medical records of 20 patients in whom FloSeal was used intraoperatively, were analyzed. Demographic data of these patients are given in [Table 1]. Intracranial surgery was performed in 17 patients while three patients underwent spinal tumour excision. Meningiomas were the most common intracranial tumours (seven patients) in whom the sealant was used. Four patients were hypertensive, two had history of coronary artery disease and one patient had diabetes nd hypothyroidism.

Median haemoglobin in the preoperative period and postoperative period was $13.15 \mathrm{~g} / \mathrm{dl}$ (range 9.3-16 g/dl) and $11.2 \mathrm{~g} / \mathrm{dl}$ (range 8.6-14.3 g/dl), respectively. Intraoperative blood loss and transfusion is shown in [Table 2]. Packed RBC transfusion was done in 15 patients and platelets and fresh frozen plasma (FFP) were used in seven patients each. Cell saver was used in six patients. Post-operatively, gross derangement in prothrombin was present in one patient. Venous infarct and haematoma occurred in one patient each while two patients developedsepsis/disseminated intravascular coagulation (DIC). Two patients were tracheostomised and two had poor outcome $(\mathrm{GOS}<4)$ at discharge.
Table 1: Demographic variables

\begin{tabular}{lc}
\hline Age (years)* & $43.1+19.6$ \\
Sex (Male: Female) & $14: 6$ \\
Weight $(\mathrm{kgs})^{*}$ & $59.2+16.8$ \\
\hline${ }^{*}$ Mean \pm SD &
\end{tabular}

*Mean \pm SD

Table 2: Intraoperative data

\begin{tabular}{lc}
\hline Variable & Mean \pm SD \\
\hline Blood loss (ml) & $1800+1518.6$ \\
Intravenous fluid transfused $(\mathrm{ml})$ & $4850+1860$ \\
Blood/blood products $(\mathrm{ml})$ & \\
RBC transfusion & $866.7+604$ \\
FFP transfusion & $728.6+485.5$ \\
PC transfusion & $312.5+120.1$ \\
\hline $\begin{array}{l}\text { RBC = Red blood cell, FFP = Fresh frozen plasma, PC = Platelet } \\
\text { concentrate, SD = Standard deivation }\end{array}$
\end{tabular}

\section{DISCUSSION}

In general, topical haemostatic agents work by active or passive promotion of coagulation protein activation and platelet adhesion, activation and aggregation. The specific mechanism of action of topical haemostatic agents varies, but includes serving as a platelet scaffold, providing exogenous factors or promoters, inhibiting fibrinolysis, dehydrating blood and causing chemical cauterisation. Typically topical agents contain collagen, cellulose, thrombin, gelatin, antifibrinolytics, minerals and/or other compounds. Fibrin sealants are derived from plasma proteins usually representing a combination of fibrinogen, fibrin-stabilising factor (factor XIII), thrombin (converts fibrinogen to fibrin) and aprotinin.

Tisseel (marketed as Hemaseel) was the first agent to receive approval of FDA in 1998. ${ }^{[2]}$ These sealants utilise vapour-heated, freeze dried pooled human fibrinogen, factor XIII, bovine aprotinin, human thrombin and calcium chloride. In 2003, Crosseal was introduced as an entirely human product that uses tranexamic acid rather than bovine aprotinin..$^{[2]}$ Although potential reactions to the bovine product are avoided, concern still exist over the use of tranexamic acid. In central nervous system, tranexamic acid may act as GABA antagonist; reason that its use in areas where it comes in contact with cerebrospinal fluid (CSF) and dura mater is contraindicated. Matrix haemostats such as FloSealutilise a special bovine collagen gelatin matrix that is cross-linked by glutaraldehyde. Like the fibrin sealants, it requires preparation with thrombin prior to application. The gelatin component of FloSeal swells up and provides mechanical compression and a basic structure for subsequent clot formation. Thrombin activates factors V, VIII, and XIII of the coagulation cascade, thus converting fibrinogen to fibrin and help 
in formation of a stable clot. It inhibits fibrinolysis and causes platelet aggregation.

Human thrombin has generally replaced bovine sources in these preparations due to acquired thrombin antibodies. Hino et al. reported three cases of iatrogenic parvovirus B19 transmission associated with commercial sealants. In swine model, intravascular bovine thrombin at a dose of $60 \mathrm{U} / \mathrm{kg}$ has been implicated for DIC and death. At lower doses $(30 \mathrm{U} / \mathrm{kg})$, thrombin causes hypotension and histologic evidence of DIC. ${ }^{[3]}$ Thrombin of bovine origin when compared to human seems to cause a greater degree of hypotension and DIC. The FDA has received four reports of improper administration of topical bovine thrombin, since 1987. Two patients were injected through dialysis access sites, one received thrombin injected directly into the spleen, and one patient was administered via nasogastric tube. Three out of these four cases died. ${ }^{[4,5]}$

History of ambulation, medical and surgical history of thrombosis, consumption of oral contraceptives and underlying hypercoaguable state should be ruled out when use of these agents is planned. Intraoperatively, sudden onset of hypotension, precipitous fall in end-tidal carbon-dioxide $\left(\mathrm{EtCO}_{2}\right)$, and sudden cardiac arrhythmias should alert the anaesthesiologist. Trans-oesophageal echocardiography (TEE) may be used to differentiate venous air embolism from topical sealant migration into vessels. TEE shows numerous mobile masses migrating from right atrium to right ventricle.

It is also important to note that with a single exposure to bovine thrombin products may lead to formation of acquired Factor $\mathrm{V}$ inhibitor. It results in the increased risk of possible coagulopathy and warrants further haematologic follow-up. ${ }^{[6]}$ The haemostatic matrices, if transferred inadvertently into the intravascular space, can cause DIC by activating the coagulation cascade and depleting fibrinogen. ${ }^{[7]}$ Sepsis/DIC occurred in two of our patients. Cell saver was used in six patients prior to FloSeal application for achieving haemostasis. In the immediate postoperative period, one patient who developed thrombocytopenia and deranged prothrombin time had suffered massive intraoperative blood loss and was subsequently transfused. So, the deranged coagulation profile cannot be attributed solely to FloSeal sealant. None of our patients who underwent spinal procedures developed any episode of postoperative haematoma. One major concern about the use of haemostatic agents in spine surgery is the danger of compression to the neural structures. FloSeal produces much less compressive effects, and has shorter time to biodegradation than the other agents. ${ }^{[8]}$ No neurological deficit or features of nerve compression were noticed in our patients.
Topical haemostatic agents have a potential to cause a severe thromboembolic event when introduced into the intravascular space. A case of fatal pulmonary embolisation of a major lung artery with FloSeal granules during removal of spinal schwanomma alerts us about occurrence of such life-threatening complications. ${ }^{[9]}$ Intraoperative anaphylaxis has also been reported following use of FloSeal. ${ }^{[10]}$ However, none of our patients experienced anaphylaxis.

FloSeal is associated with a significantly shortened hospital stay and duration of pain-medication use and overall reduction in consumption/demand in adults undergoing tonsillectomy. ${ }^{[11]}$ It has been used to stop persistent intraoperative bleeding during Caesarean section, ${ }^{[12]}$ in severe postpartum haemorrhage, during gynaecological laparoscopy. ${ }^{[13]}$ It can be used safely in head and neck surgeries without any adverse effects. ${ }^{[14]}$ FloSeal is quite expensive, hence, its use is more logical in major surgical procedures and acute life-threatening haemorrhages. However, its application is valuable in some moderately critical patients with severe concomitant diseases and coagulation disorders to avoid long hospital stays, longer immobilisation, and higher cost of in-hospital care.

\section{CONCLUSIONS}

FloSeal is a safe and efficacious adjunct to stop intraoperative bleeding during neurosurgical procedures. It provides effective and immediate haemostasis during neurosurgery by preventing blood loss, vessel compromise, cerebral ischaemia, permanent neurological squeal or even death. It is also important in neuroendoscopic procedures where a mild bleeding may obscure the vision. We conclude that though the use of FloSeal is safe in neurosurgical practice, the possible complications due to this agent should be borne in mind during intraoperative administration.

\section{REFERENCES}

1. $\mathrm{Oz}$ MC, Rondinone JF, Shargill NS. Floseal Matrix: New generation topical hemostatic sealant. J Card Surg 2003;18:486-93.

2. Spotnitz WD, Burks S. State-of-the-Art Review: Hemostats, sealants, and adhesives II: Update as well as how and when to use the components of the surgical toolbox. Clin Appl Thromb Hemost 2010;16:497-514.

3. Pusateri AE, Holcomb JB, Bhattacharyya SN, Harris RA, Gomez RR, MacPhee MJ, et al.Differenthypotensiveresponses to intravenous bovine and human thrombin preparations in swine. J Trauma 2001;50:83-90.

4. Gabay M. Absorbable hemostatic agents. Am J Health Syst Pharm 2006;63:1244-53.

5. Gershon SK,Chang AC,Purvis WV,Salive M.Misadministration of topical bovine thrombin. JAMA 1999;282:1919.

6. Kajitani M, Ozdemir A, Aguinaga M, Jazieh AR, Flick JT, Antakli T. Severe hemorrhagic complication due to acquired 
factor $\mathrm{V}$ inhibitor after single exposure to bovine thrombin product. J Card Surg 2000;15:378-82.

7. Levi M. Disseminated intravascular coagulation. Crit Care Med 2007;35:2191-5.

8. Yao HH, Hong MK, Drummond KJ. Haemostasis in neurosurgery: What is the evidence for gelatin-thrombin matrix sealant? J Clin Neurosci 2013;20:349-56.

9. Steinestel K, Geiger A, Naraghi R, Kunz U, Danz B, Kraft K, et al. Fatal thromboembolism to the left pulmonary artery by locally applied hemostatic matrix after surgical removal of spinal schwannoma: A case report. Hum Pathol 2013;44:294-8.

10. Spencer HT, Hsu JT, McDonald DR, Karlin LI. Intraoperative anaphylaxis to gelatin in topical hemostatic agents during anterior spinal fusion: A case report. Spine J 2012;12:e1-6.

11. Mozet C, Prettin C, Dietze M, Fickweiler U, Dietz A. Use of Floseal and effects on wound healing and pain in adults undergoing tonsillectomy: Randomised comparison versus electrocautery. Eur Arch Otorhinolaryngol 2012;269:2247-54

12. Lemmer R, Albrech M, Bauer G. Use of FloSeal hemostatic matrix in a patient with severe postpartum hemorrhage. J Obstet Gynaecol Res 2012;38:435-7.

13. Gorry A, Morelli ML, Olowu O, Shahid A, Odejinmi F. Laparoscopic management of abdominal ectopic pregnancy using FLOSEAL Hemostatic Matrix. Int J Gynaecol Obstet 2012;117:83-4.

14. Ujam A, Awad Z, Wong G, Tatla T, Farrell R. Safety trial of Floseal haemostatic agent in head and neck surgery. Ann R Coll Surg Engl 2012;94:336-9.

How to cite this article: Khanna P, Mahajan C, Gupta P, Banik S, Ray BR, Rath GP. Use of gelatin-thrombin matrix haemostatic sealant in neurosurgery: Anaesthetic implications and review of literature. J Neuroanaesthesiol Crit Care 2015;2:51-4.

Source of Support: Nil, Conflict of Interest: None declared.

\section{Staying in touch with the journal}

1) Table of Contents (TOC) email alert Receive an email alert containing the TOC when a new complete issue of the journal is made available online. To register for TOC alerts go to www.ijem.in/signup.asp.

\section{2) RSS feeds}

Really Simple Syndication (RSS) helps you to get alerts on new publication right on your desktop without going to the journal's website. You need a software (e.g. RSSReader, Feed Demon, FeedReader, My Yahoo!, NewsGator and NewzCrawler) to get advantage of this tool. RSS feeds can also be read through FireFox or Microsoft Outlook 2007. Once any of these small (and mostly free) software is installed, add www.ijem.in/rssfeed.asp as one of the feeds. 